\title{
Diagnostic utility of the Lymphoid Screening Tube for Ogata score calculation in MDS investigation
}

\section{OBJECTIVES}

The diagnosis of myelodysplastic syndrome (MDS) is not always straightforward in the absence of objective markers such as ringed sideroblasts, an excess of blasts or clonal cytogenetic abnormalities. Moreover, the lack of specificity of dysplasia makes the differentiation with other causes of peripheral cytopenia difficult. The WHO 2016 classification of MDS recognizes multiparameter flow cytometry (MFC) as an adjuvant tool for MDS diagnosis. An easily applicable MFC protocol based on CD34 and CD45 is proposed by Ogata et al. Furthermore, in the diagnostic workup of patients with peripheral cytopenia, MFC by means of a Lymphoid Screening Tube (LST) is recommended by the EuroFlow ${ }^{T M}$ consortium.

The aim of this study was to investigate whether the LST, supplemented with CD34, can be used to calculate the Ogata score.

\section{METHODS}

\section{Samples}

All bone marrow samples $(n=108)$ received between January 2016 and December 2016 for investigation of peripheral blood cytopenia were analyzed:

- $\operatorname{MDS}(\mathrm{n}=32)$

- non-MDS $(\mathrm{n}=76)$

Table 1. Ogata score calculation.

\begin{tabular}{lrr}
\hline FC parameter & Cut-off & Score \\
1. Myeloblasts in all CD45+ cells (\%) & $\geq 2$ & 1 \\
2. Lymphoblasts in all CD34+ cells (\%) & $\leq 5$ & 1 \\
3. Lymphocytes $\div$ myeloblasts MFI of CD45 ratio & $\leq 4$ or $\geq 7.5$ & 1 \\
4. Granulocytes $\div$ lymphocytes SSC ratio & $\leq 6$ & 1 \\
\hline
\end{tabular}

\section{Methods}

The Ogata score is considered positive, and thus suggestive of MDS, if a value of 2 or more is observed. Results of the Ogata score were compared with the clinical diagnosis according to the WHO criteria, based on extensive history taking, cytomorphology and cytogenetics, but not on flowcytometric data.
The Ogata score was calculated with an LST based on the tube designed by the EuroFlow ${ }^{\mathrm{TM}}$ consortium, but with addition of CD34 and without TCR $\gamma \delta$.

\begin{tabular}{|c|c|c|}
\hline Fluorochrome & EuroFlow $^{\text {TM }}$ & AZ Sint-Jan \\
\hline HV450 & CD20 & CD20 \\
\hline HV450 & CD4 & CD4 \\
\hline HV500 & CD45 & CD45 \\
\hline FITC & CD8 & $\mathrm{CD} 8$ \\
\hline FITC & Lambda & Lambda \\
\hline PE & Kappa & Kappa \\
\hline PE & CD56 & CD56 \\
\hline PerCP-Cy5.5 & CD5 & CD5 \\
\hline PerCP-Сy5.5 & - & CD34 \\
\hline PE-Cy7 & $\mathrm{TCR} \gamma / \delta$ & - \\
\hline PE-Cy7 & CD19 & CD19 \\
\hline APC & CD3 & CD3 \\
\hline APC-H7 & CD38 & CD38 \\
\hline
\end{tabular}

\section{RESULTS}

Table 3. Patient characteristics and microscopic evaluation of dysplasia.

\begin{tabular}{rrrrr}
$\begin{array}{r}\text { Number } \\
\text { (M/F) }\end{array}$ & $\begin{array}{r}\text { Mean age } \\
\text { (range) }\end{array}$ & $\begin{array}{c}\text { Abnormal } \\
\text { karyotype }\end{array}$ & \multicolumn{2}{c}{$\begin{array}{c}\text { Microscopic dysplasia } \\
\text { evaluation }\end{array}$} \\
\cline { 3 - 6 } & & & significant & $\begin{array}{c}\text { in- } \\
\text { significant }\end{array}$
\end{tabular}

\section{$\operatorname{MDS}(\mathrm{n}=32)$}

Low-grade $(n=22)$

SLD $\quad 4(4 / 0) \quad 70(65-79) \quad 3(75 \%)$

MLD $\quad 13(9 / 4) \quad 72(46-88) \quad 5(38 \%)$

RS-SLD $1(1 / 0)$

RS-MLD $1(0 / 1)$

$5 q-\quad 3(1 / 2) \quad 79(73-83) \quad 3(100 \%)$

High-grade $(n=10)$

$\begin{array}{llll}\text { EB-1 } & 4(1 / 3) & 71(49-85) & 2(50 \%)\end{array}$

EB-2 $\quad 6(3 / 3) \quad 68(57-78) \quad 2(40 \%)$

Non-MDS $(n=76)$

Insignificant (=doubtful) morphological dysplasia was found

in 19 cases, impeding diagnostic interpretation. In this group

a true positive Ogata score was found in 4/6 MDS cases

a true negative score was found in $13 / 13$ non-MDS cases
Table 4. Results Ogata score.

\begin{tabular}{|c|c|c|c|c|c|c|c|c|}
\hline & \multicolumn{5}{|c|}{ Ogata score } & \multirow[b]{3}{*}{$\begin{array}{r}\text { Positive } \\
\text { score }\end{array}$} & \multirow[b]{3}{*}{ Sensitivity } & \multirow[b]{3}{*}{ Specificity } \\
\hline & - & & + & & & & & \\
\hline & 0 & 1 & 2 & 3 & 4 & & & \\
\hline MDS $(n=32)$ & & & & & & $20 / 32$ & $63 \%$ & NA \\
\hline Low-grade $(n=22)$ & & & & & & $12 / 22$ & $55 \%$ & $N A$ \\
\hline SLD & 0 & 2 & 2 & 0 & 0 & $2 / 4$ & $50 \%$ & NA \\
\hline MLD & 5 & 1 & 4 & 3 & 0 & $7 / 13$ & $54 \%$ & NA \\
\hline RS-SLD & 0 & 0 & 1 & 0 & 0 & $1 / 1$ & $100 \%$ & NA \\
\hline RS-MLD & 1 & 0 & 0 & 0 & 0 & $0 / 1$ & $0 \%$ & NA \\
\hline $5 q-$ & 1 & 0 & 1 & 1 & 0 & $2 / 3$ & $67 \%$ & NA \\
\hline High-grade $(n=10)$ & & & & & & $8 / 10$ & $80 \%$ & $N A$ \\
\hline EB-1 & 0 & 1 & 1 & 2 & 0 & $3 / 4$ & $75 \%$ & NA \\
\hline EB-2 & 0 & 1 & 1 & 2 & 2 & $5 / 6$ & $83 \%$ & NA \\
\hline Non-MDS $(n=76)$ & & & & & & $6 / 76$ & NA & $92 \%$ \\
\hline
\end{tabular}

Sensitivity

- MDS: $63 \%$

Specificity

low-grade MDS: $55 \%$

high-grade MDS: $80 \%$

\section{CONCLUSION}

Besides screening for clonal lymphocytes, plasma cells and blasts, an LST supplemented with CD34 allows the calculation of the Ogata score as an adjuvant tool in the diagnostic workup of cytopenic patients suspected of MDS. 